\title{
0 lúdico e 0 ensino de Geociências no Brasil: principais tendências das publicações na área de Ciências da Natureza
}

The ludic and Geosciences teaching in Brazil: main trends of the publications in the area of Natural Sciences

\author{
Danilo Missias Teixeiraª , Fábio Braz Machado², Josilaine Santina da Silva ${ }^{3}$
}

1- Doutorando, Progr. Pós-Grad. Ensino e História de Ciências da Terra, Unicamp. Rod. 020 km 02, Vila Rosário, Correntina-BA. - danquimica 2010@gmail.com

2- Prof. Adjunto IV, Depto. de Clências Ambientals, Univ. Fed. São Paulo. Rua Antônio Doll de Moraes, 105 Centro, Diadema, SP. - fabio.machado@unifesp.com

3- Mestranda Progr. Pós-Grad. Ensino e História de Ciências da Terra, Unicamp. End.: Rua Coruripe, nº 01-Canaã, Maceí/AL. - josy_hoje@hotmail.com

ABSTRACT: One of the methodologies of teaching in the Teaching of Sciences are ludic activities, which furnish a study of concepts in a more dynamic and interactive form. However, when it comes to the Geosciences concepts, there is a difficulty exhibited by teachers of Exact and Biological Sciences, which prevents these professionals from elaborating proposals related to these concepts. Thus, the objective of this research is to identify the main trends of works with playful characteristics that focus on Geosciences concepts in the scientific literature of Science Teaching. In order to accomplish this, a search was made in the 1) Thesis in CAPES (Coordenação de Aperfeiçoamento de Pessoal de Nível Superior) database, and in 2) periodicals related to Teaching of Sciences. It was possible to observe that ludic publications in Science Teaching exhibit the following trends: 1) Most publications originate in Southeast Brazil, mainly in the states of Rio de Janeiro and São Paulo, 2) The number of publications is increasing since 2014, with a high in 2016, 3) the area of knowledge presenting the most works is Chemistry, followed by Biology. 4) these publications are mostly experiences applied in high school, and 5) the most frequent play mode is Didactic Game.

\section{Manuscrito:}

Recebido: $12 / 09 / 17$

Corrigido: $30 / 10 / 17$

Aceito: $22 / 11 / 17$

Citation: Teixeira D.M., Machado F.B., Silva J.S. 2017. 0 lúdico e 0 ensino de Geociências no Brasil: principais tendências das publicações na área de Ciências da Natureza. Terræ Didatica, 13(3):286-294. < http://www.ige. unicamp.br/terraedidatica/>.

Keywords: Ludic, Geosciences, Science Teaching.

\section{Introdução}

Um dos maiores desafios do Ensino de Ciências é propor maneiras de despertar o interesse dos estudantes da Educação Básica (Ensino Fundamental e Médio) pelo conhecimento científico, uma vez que os conceitos ligados aos conteúdos das disciplinas de Ciências (Química, Física e Biologia) são tidos como pouco atrativos aos alunos ou de difícil compreensão. Isso ocorre por diversos motivos, dentre os quais destacam-se: 1 ) a maneira como os conteúdos são abordados nas aulas (Fourez 2003); 2) o método utilizado pelo professor durante a exposição do conteúdo em sala (Veiga 2006).

O primeiro motivo está ligado a abordagem do conteúdo em suas aulas, ou seja, como os conceitos são apresentados e como o estudo é conduzido entre professor e estudantes. Já o segundo motivo se refere aos artifícios utilizados pelo professor e/ ou caminho traçado para atingir o aprendizado. Para promover um ensino de Ciências que envolva contribuições de professores e alunos, é necessário que ele faça sentido no universo dos estudantes. Assim, é preciso valorizar o contexto social em que os estudantes estão inseridos, promovendo um diálogo entre os conceitos científicos e as situações vivenciadas no seu cotidiano. Sobre essa questão, Fourez (2003, p.110) aponta que:

Os alunos teriam a impressão de que se quer obrigá-los a ver o mundo com os olhos de cientistas. Enquanto o que teria sentido para eles seria um ensino de Ciências que ajudasse a compreender o mundo deles. Isto não quer dizer, absolutamente, que gostariam de permanecer em seu pequeno universo; mas, para que tenham sentido para eles os modelos científicos cujo estudo thes é imposto, estes modelos deveriam permitirlhes compreender a "sua" história e o "seu" mundo. Ou seja: os jovens prefeririam cursos de ciências que não sejam centrados sobre os interesses de outros (quer seja a comunidade de cientistas ou o mundo industrial), mas sobre os deles próprios.

Nesse sentido, o que se busca com o ensino de Ciências não é a formação de cientistas, mas 
de indivíduos capazes de questionar, interferir e modificar a realidade na qual estão inseridos, por meio do conhecimento científico, adquirido nas aulas. Esse conhecimento será o ponto de partida para suas reflexões pessoais e profissionais, dando-lhes subsídios para discutir assuntos políticos, econômicos e sociais.

Torna-se então necessário que o professor encontre caminhos possíveis para este tipo de abordagem (valorização do contexto do aluno). Para isso, diferentes estratégias - metodologias, ferramentas e instrumentos de ensino - podem ser utilizadas para que os conceitos científicos sejam mais bem compreendidos pelos estudantes relacionando-se, portanto, com o método utilizado pelo professor (segundo motivo destacado anteriormente).

Para Veiga (2006) o professor precisa estar solicito às mudanças em seu método de ensino, uma vez que as constantes transformações que ocorrem na sociedade proporcionam novas abordagens para um mesmo conteúdo. Com isso, o professor assume o seu papel de mediador no processo de construção do conhecimento.

Várias são as metodologias de ensino-aprendizagem apontadas na literatura científica do Ensino de Ciências, das quais podem ser citadas a "Experimentação" (Ferreira et al. 2010) (Guimarães 2009), as "Atividades Lúdicas" (Kishimoto 2007, Rau 2011, Soares 2013), as "Tecnologias Educacionais" (Luckesi 1982, Villardi \& Oliveira 2005), dentre outras.

As "Atividades Lúdicas" vêm ganhando espaço na comunidade científica, principalmente na última década. O uso do lúdico como ferramenta de ensino e aprendizagem vem sendo estudado desde os anos 1990. O fato de poder trabalhar os conceitos científicos de uma forma mais "descontraída" e diferenciada tem servido de incentivo para um número crescente de professores fazerem uso dela. Isso aumenta o interesse pela pesquisa no Ensino de Ciências, em buscar caracterizar, compreender e divulgar o uso do lúdico no contexto educacional.

Para Benvenutti et al. (2010) o lúdico faz parte das ações humanas, possibilitando resgates sociais e culturais e, quando atrelado à fins didáticos, ganha um formato de ação pedagógica, ou seja, uma atividade que visa o ensino e o aprendizado. Assim, como ação humana, o lúdico pode ser uma importante metodologia de ensino para uma abordagem contextualizada dos conteúdos.
Quando se trata de publicações de Ensino de Ciências, existe carência de publicações que apresentem propostas relacionadas a conteúdos de característica lúdica, em que os conceitos básicos de Geociências sejam contemplados. De acordo com os Parâmetros Curriculares Nacionais para o Ensino Fundamental (PCN), os conceitos definidos para a disciplina de Ciências Naturais, incluem - Tempo Geológico, Estrutura da Terra, Ciclo das Rochas e Minerais - os quais devem ser abordados entre o $6^{\circ}$ e o $9^{\circ}$ ano. Apesar da carência de publicações, o uso do lúdico no Ensino de Ciências vem apresentando um crescimento nas últimas décadas. Os professores começaram a aceitar e utilizar esta metodologia de ensino, baseando-se em resultados de pesquisas e relatos de experiências apresentados nas publicações da área. Assim, torna-se necessário saber como tem sidotais publicações, quais suas principais abordagens e conteúdo temático.

Nesse sentido, este artigo tem como principal objetivo identificar as principais tendências de trabalhos com características lúdicas que possuam como foco os conceitos de Geociências. Isso será feito utilizando publicações vinculadas ao Ensino de Ciências, já que a ideia é observar como evoluem as tendências na área de Ciências da Natureza.

\section{Metodologia}

Busca-se identificar as principais tendências apresentadas na literatura científica do Ensino de Ciências, que tenham como foco as Atividades Lúdicas, nas mais diversas manifestações (jogos, brincadeiras e brinquedos) ou que discutam o lúdico de maneira geral, de forma teórica ou aplicada.

Sendo o objetivo identificar as tendências sobre o lúdico nas últimas décadas, por isso, foi adotado o período dos anos de 2000 a 2016, onde se utilizou as palavras-chaves: lúdico, ludicidade, jogos, brincadeiras. Assim, o trabalho partiu de uma busca no Banco de Teses e Dissertações da Coordenação de Aperfeiçoamento de Pessoal de Nível Superior (CAPES), além de leitura de artigos publicados em Periódicos vinculados ao "Ensino de Ciências", que apresentam Qualis B2 até-A1.

Os trabalhos identificados pela busca foram selecionados, tabulados e descartados aqueles que não apresentavam relação direta com o objetivo do trabalho. Foi feita uma análise detalhada para os trabalhos que apresentam foco do lúdico no ensino de Geociências, uma vez que, este é o principal propósito deste artigo. 


\section{Resultados e discussão}

A identificação no Banco de Teses e Dissertações da CAPES ocorreu por barra de busca online, aplicando-se inicialmente o filtro ano de publicação, ao qual se selecionou o intervalo 2000 a 2016. Após a busca utilizando todas as palavras-chave foram encontradas 2856 publicações (entre teses e dissertações) de todas as áreas. Considerando que o foco da pesquisa é o Ensino de Ciências, foi necessária a aplicação de mais um do filtro de busca on line, a área temática selecionando-se as áreas de Química, Física, Biologia, Geociências e Ensino de Ciências. Com isso o número de publicações identificadas reduziu para 213 (187 dissertações de Mestrado e 26 teses de Doutorado), 7,5\% do total encontrado para todas as áreas de conhecimento.

O levantamento dos periódicos vinculados ao Ensino de Ciências teve como parâmetros o Qualis B2, B1, A2 e A1 para o ano de 2016, na área de Ciências Exatas, verificado no site da CAPES. Foram analisados 36 periódicos e identificados 136 artigos publicados no período de 2000 a 2016.

A partir dos dois levantamentos foram identificados 349 trabalhos que utilizam o lúdico no Ensino de Ciências. A Fig. 1 apresenta a distribuição dos trabalhos publicados durante o intervalo de anos selecionado.

Trabalhos identificados por ano de publicação

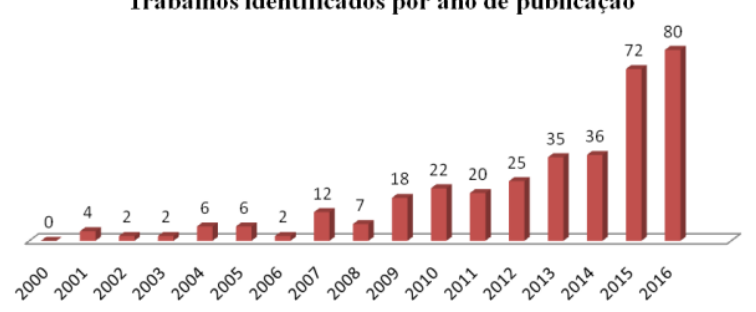

Figura 1. Distribuição de trabalhos identificados no levantamento e seus respectivos anos de publicação utilizando as palavras chaves lúdico, ludicidade, jogos, brincadeiras

Ainda, de acordo com a Fig. 1, o intervalo compreendido entre 2014 e 2016 apresenta maior número de publicações, fato que pode ser justificado a partir devários acontecimentos:

1- O XVI Encontro Nacional de Ensino de Química (ENEQ), realizado em 2012 em Salvador/BA, durante o qual ocorreram três minicursos sobre o lúdico - "Experimentação lúdica e investigativa com o uso de material alternativo", "Jogos e brincadeiras no ensino de Química" e "O RPG (Role Playing Game) como estratégia de Problematização e Avaliação no Ensino de Química”. O evento contou com a participação de pesquisadores de todo o país, sendo que os mini-cursos representam uma forma interessante de divulgar o lúdico, o que pode ter influenciado no aumento do número de publicações;

2- A publicação em 2013 do livro "Jogos e Atividades Lúdicas para o Ensino de Química”, do professor Marlon Soares (Universidade Federal de Goiás), que trouxe discussões teóricas e filosóficas sobre o lúdico, bem como sua utilização no contexto do Ensino de Ciências, mais precisamente, no Ensino de Química;

3- O $1^{\circ}$ Encontro Nacional de Jogos e Atividades Lúdicas no Ensino de Química (JALEQUIM), ocorrido em 2014 na Universidade Federal de Goiás, que contou com mais de 200 participantes oriundos de 17 estados brasileiros.

OXX Simpósio Nacional de Ensino de Física, realizado em 2013 em São Paulo/SP contou com duas oficinas com abordagens lúdicas: "Ensino de Física com Brinquedos Científicos"; o "Roleplaying Game (RPG) como estratégia didática: Jogando Um RPG sobre Fontes Renováveis de Energia”; e o minicurso "Algumas estratégias para o uso da Aprendizagem Ativa no Ensino da Física". Ainda, em 2012 durante o $46^{\circ}$ Congresso Brasileiro de Geologia, realizado em Santos/SP pela Sociedade Brasileira de Geologia, foi organizado sobre a areia da praia do Gonzaga (importante praia da cidade) a atividade "Praia das Geociências" que, durante 7 dias, atendeu mais de 15 mil pessoas (principalmente crianças) com atividades lúdicas relacionadas com Geologia como jogos de dados, oficina de réplica de fósseis, leitura de mapas, formação de solo e distribuição de amostras de petróleo do pré-sal para cada visitante. As referências sobre as atividades ainda podem ser facilmente localizadas em vídeos publicados na internet tais como https:// www.youtube.com/watch?v=_PNSw9Laz9E ou http://g1.globo.com/sp/santos-regiao/noticia/2012/10/fezes-de-dinossauros-fossilizadas-levam-curiosos-praia-em-santos.html .

Estes eventos, assim como outros vinculados ao ensino de ciências, podem ter proporcionado momentos de conhecimento do lúdico como estratégia de ensino, o que levou ao aumento do número de teses e dissertações a partir do ano de 2013. Além disso, o número de artigos publicados 
pode ter sido diretamente influenciado pelas teses e dissertações defendidas.

A Fig. 2 apresenta a distribuição dos trabalhos considerando os estados brasileiros aos quais pertencem as instituições de ensino e pesquisa que estão vinculados aos pesquisadores responsáveis pelos trabalhos identificados.

O gráfico da Fig. 2 mostra que a região Sudeste apresenta um total de 171 publicações, o que representa $49 \%$ do total de trabalhos e que o Rio de Janeiro foi o estado que originou maior número de publicações identificadas, seguido por São Paulo.

A região Sudeste contempla grandes universidades brasileiras, como a Universidade de São Paulo (USP), Universidade Estadual de Campinas (Unicamp), Universidade Federal de São Paulo (UNIFESP), Universidade Estadual Paulista (UNESP), Universidade Federal de São Carlos (UFSCAR), Universidade Federal do Rio de Janeiro (UFRJ), Universidade Federal Fluminense (UFF), dentre outras. Isso explica o grande número de publicações nessa região, uma vez que, muitos programas de pós-graduação (ou até mesmo apenas graduação) vinculados ao Ensino de Ciências são ofertados nessas instituições.

Após Rio de Janeiro e São Paulo, Goiás apresentou o maior número de publicações identificadas no levantamento, posição justificada pela presença do Laboratório de Educação Química e Atividades Lúdicas (LEQUAL) na Universidade Federal de Goiás, cujo objetivo principal é o desenvolvimento de propostas lúdicas de fácil aplicação em sala de aula e outras pesquisas vinculadas ao Ensino de Química.

Em relação à categoria modalidade de ensino (Fig. 3), percebe-se que o ensino médio apresenta 44 publicações (41\%), maior número de publicações vinculadas como resultado da pesquisa elaborada.Torna-se necessário ressaltar que, algumas publicações são fruto de trabalhos em uma ou mais modalidades de ensino, ou seja, a mesma publicação pode ter sido oriunda de experiências em mais de uma modalidade de ensino ao mesmo tempo.

A concentração das atividades no ensino médio pode ser justificada pelo fato de algumas disciplinas, como Física e Química, serem ofertadas a partir desta etapa da educação básica. Até o $8^{\circ}$ ano do Ensino Fundamental II, os alunos aprendem os conceitos básicos do Ensino de Ciências, por meio da disciplina Ciências Naturais, sendo que, a partir do $9^{\circ}$ ano a disciplina é fragmentada em três outras: Biologia; Química; e Física.

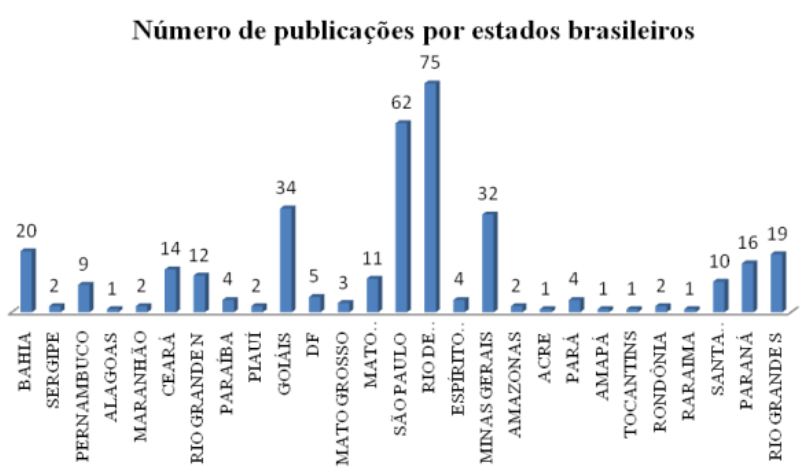

Figura 2. Distribuição das publicações, identificadas por estados brasileiros, no intervalo considerado na pesquisa

Por sua vez o Ensino Fundamental II, que corresponde à disciplina de Ciências Naturais $\left(6^{\circ}\right.$ a $9^{\circ}$ ano), apresenta apenas 53 publicações (15\%). Esse número é menor quando comparado ao do Ensino Médio, uma vez que, a faixa etária dos estudantes do Fundamental II é bem menor, o que poderia ser um forte argumento para utilização de atividades lúdicas. Além disso, observa-se também que os artigos vinculados ao ensino superior somam apenas $11 \%$ do total, o que pode ser justificado pelas publicações de professores das universidades referentes às experiências em sala de aula dos cursos acadêmicos. Teixeira (2016) destacam a importância de desenvolver trabalhos lúdicos nos Cursos de Licenciatura, onde os estudantes podem desenvolver características pessoais, (habilidade manual, criatividade e coletividade) e profissionais (didática, planejamento de aula, estudo do conteúdo científico e desenvolvimento do interesse de alunos). Segundo os autores, é importante para o futuro professor conhecer a metodologia lúdica e utilizá-la em suas aulas.

Número de publicações por modalidade de ensino

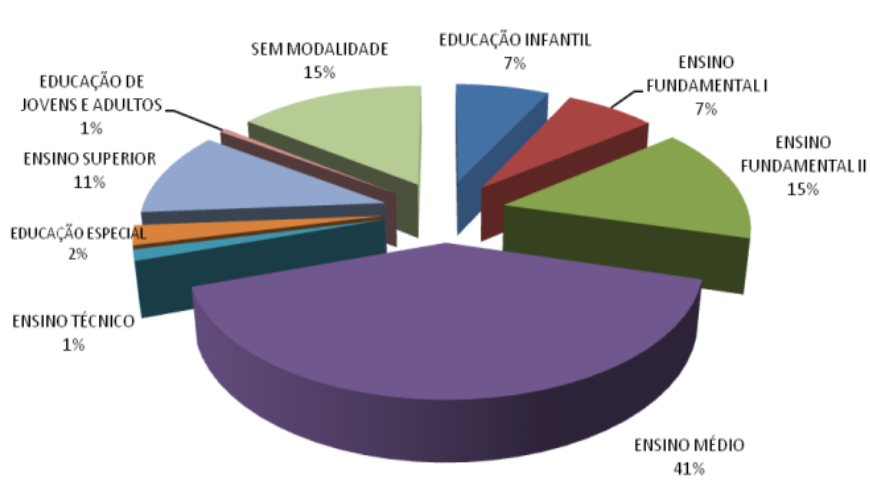

Figura 3. Distribuição das publicações segundo a modalidade de ensino no intervalo considerado na pesquisa. Numero total de publicações é de 349 trabalhos 
Por fim, as publicações sem modalidade de ensino representam $15 \%$ do total de trabalhos identificados. São aquelas não direcionadas para a escola, ou seja, trata-se de pesquisas teóricas sobre o lúdico entre as quais destaca-se a tese de Oliveira (2009) - "Atividades de experimentação investigativas lúdicas no ensino de química: um estudo de caso" - na qual o autor apresenta uma reflexão sobre as potencialidades da Experimentação como um recurso lúdico a ser utilizado em sala de aula, mas não se refere a uma modalidade de ensino específica. Trata-se então, de uma pesquisa teórica, mas sem modalidade de ensino definida.

Outro item analisado foi a área de conhecimento a qual pertenciam as publicações identificadas. A Fig. 4 identifica as áreas de conhecimentos e seus respectivos números de publicações. Torna-se necessário ressaltar que as publicações oriundas de experiências ou pesquisas da Educação Infantil foram enquadradas na categoria pedagogia.

Percebe-se que Biologia e a Química são as áreas de conhecimento que apresentam maior número de Teses e Dissertações no Ensino de Ciências com foco no Lúdico. As duas áreas representam cerca de $48 \%$ das publicações identificadas durante o levantamento.

Os minicursos e oficinas sobre o lúdico nos eventos do Ensino de Biologia e Química podem ter influenciado o aumento no número de publicações. Foram identificados trabalhos ligados à Educação Ambiental que contemplam temas como Ecologia, Poluição (ar, água e solo), Conservação dos Ambientes Naturais, e Sustentabilidade.

Os trabalhos vinculados à Biologia (ensino médio) foram dissociados daqueles oriundos da disciplina de Ciências (ensino fundamental), uma vez que representam trabalhos de profissionais diferentes. Torna-se possível que, para o ensino médio, na disciplina de Biologia, apenas profissionais desta área produziram estes trabalhos, enquanto nas Ciências do ensino fundamental profissionais de Biologia, Química e Física podem ter atuado nas publicações.

Ainda, outro fato observado e que apresenta forte relação com a pesquisa, foi o pequeno número de trabalhos vinculados ao ensino de Geociências. Foram apenas 07 (2\%), o que demonstra carência de propostas lúdicas nesta área. Para Carneiro et al. (2004), existem várias razões para propor atividades vinculadas à Geociências na educação básica, dentre as quais, a necessidade de trabalhar questões de Sustentabilidade e uso

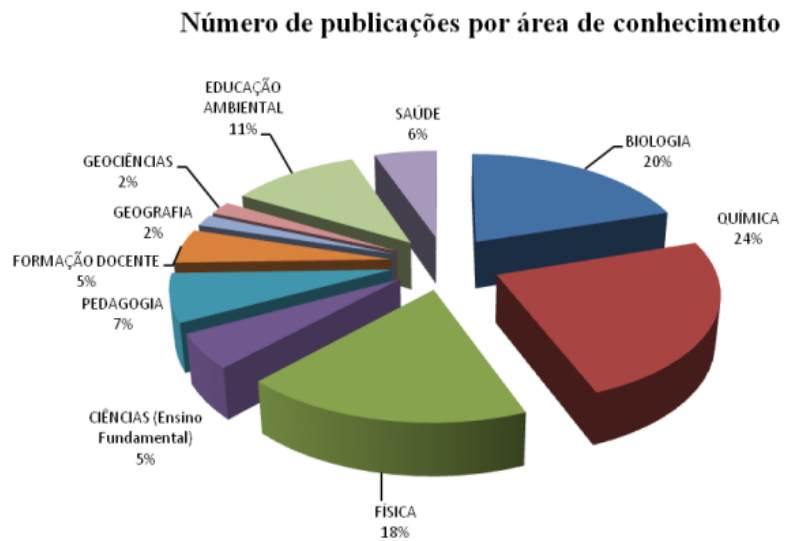

Figura 4. Relação entre o número de publicações identificadas e as áreas de conhecimento no intervalo considerado na pesquisa. Número total de publicações é de 349 trabalhos

de recursos naturais de forma adequada, além de entender as características e utilidades dos aspectos contidos no Sistema Terra.

Há carência de propostas para conteúdos ligados a Geociências não somente com características lúdicas, mas de diferentes naturezas metodológicas. Lopes \& Carneiro (2009) defendem o o uso do lúdico como uma ferramenta interessante onde a a maioria dos professores considera a introdução do lúdico como estratégia de ensino adequada às novas exigências do ensino, valorizando a utilização pedagógica de recursos como os jogos didáticos.

Assim, utilizar propostas lúdicas vinculadas ao ensino de Geociências seria uma iniciativa que proporciona um melhor aprendizado dos conceitos ligados a essa área, o que é importante para o desenvolvimento de um senso crítico no estudante, principalmente em questões ligadas aos conceitos de Recursos Naturais e Meio Ambiente.

Por fim, buscou-se conhecer quais as formas lúdicas apresentadas nessas publicações, os artifícios (ou manifestações) do lúdico que os autores escolheram para suas pesquisas. A Fig. 5 apresenta as formas lúdicas e os respectivos números de publicações em que aparecem.

A Fig. 5 mostra que o Jogo Didático é a atividade lúdica mais abordada nas publicações identificadas. Para Rau (2011), é mais utilizado por ser a ferramenta lúdica mais conhecida, uma vez que é mais fácil adaptar jogos tradicionais do que pensar em brincadeiras ou dinâmicas novas. As Brincadeiras (segunda atividade lúdica mais encontrada nos trabalhos) são muito utilizadas 


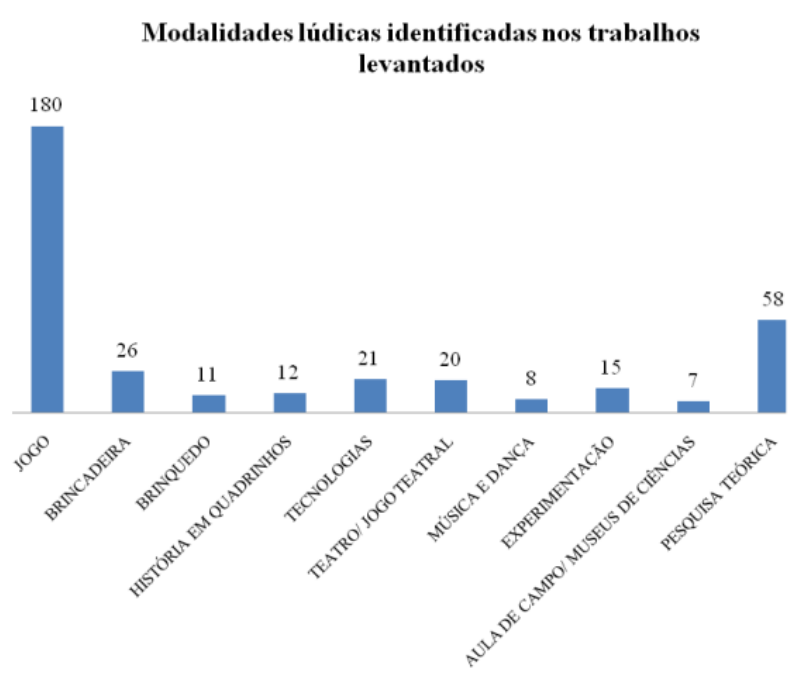

Figura 5. Relação entre o número de trabalhos identificados e as modalidades lúdicas utilizadas pelos autores no intervalo considerado na pesquisa

na educação infantil, para trabalhar a coordenação motora, aspectos sociais e de coletivismo. No levantamento percebemos trabalhos de Física que faziam uso desse recurso, principalmente articulado com Experimentos ou Tecnologias de Informação e Comunicação (TIC's).

As Pesquisas Teóricas que abordavam conceito, origem ou propostas lúdicas (sem aplicação), representam cerca de $30 \%$ das abordagens nas publicações levantadas. Isso pode indicar que os pesquisadores lúdicos do ensino de ciências estão se preocupando em trazer definições e conceitos fundamentais para delimitar melhor esta metodologia.

Após o levantamento, identificação dos trabalhos, categorização e breve discussão, foi possível observar que as publicações lúdicas no ensino de ciências apresentam as seguintes tendências:

1 - A maior parte das publicações é produzida na região sudeste do país, principalmente nos estados de Rio de Janeiro e São Paulo;

2- O número de publicações é crescente a partir do ano de 2014, com maior índice verificado em 2016;

3- Química, seguida por Biologia, apresentaram maior concentração de trabalhos por área de conhecimento;

4- As publicações são, em sua maior proporção, experiências realizadas no Ensino Médio, com a modalidade lúdica Jogo Didático.

\section{Publicações lúdicas e 0 ensino de Geociências}

A Fig. 4 mostra os trabalhos identificados no levantamento, com foco voltado para Geociências , apenas sete trabalhos (de um total de 349) tem como foco o lúdico no Ensino de Ciências para estudo de conceitos de Geologia, número que mostra a carência de trabalhos lúdicos para área. Assim, é possível perceber que os educadores (professores e pesquisadores da Educação) têm limitações em relação a propostas de ensino (testadas, analisadas e publicadas) para serem utilizadas em suas aulas.

A Tab. 1 apresenta os trabalhos identificados com o tema geociências no intervalo considerado na pesquisa.

Considerando que os trabalhos listados na Tab. 1 apresentam ideias vinculadas ao Ensino de Geociências de forma direta (como no caso dos títulos) e de forma indireta (quando ao longo do texto mostram articulações da área), é importante e necessário estabelecer a definição de Geociências.

Na perspectiva de Potapova (1968), as Geociências compreendem o estudo da Natureza, seus fenômenos físicos, químicos e biológicos, e as interações dos seres vivos com os não vivos. Nesse sentido, o termo Geociências compreende também os fenômenos geológicos, onde a Geologia poderia ser compreendida como sinônimo (ou complemento) das Geociências.

Para Frodeman (1995), as Geociências podem ser entendidas como Ciências da Terra - o que se articula com a ideia apresentada por Potapova (1968); isso torna possível entender a fundo as questões vinculadas à realidade pública, social e da natureza, ou seja, parte-se de uma análise histórica, epistemológica e física dos fenômenos. Para isso, faz uso de ferramentas das Ciências Naturais (Física, Química e Biologia) e das Humanas, como Geografia e História.

Nesse sentido, pode-se sugerir uma definição própria para Geociências, combinando as afirmações de Potapova (1968) e Frodeman (1995, 2010), em que ela seria a área de conhecimento que estuda os fenômenos naturais sob um olhar físico, histórico e epistemológico, fazendo uso da perspectiva interdisciplinar (interação entre as disciplinas) para tal estudo. A partir dessa definição, analisam-se os trabalhos identificados, interagindo a metodologia lúdica com os conceitos de Geociências.

O trabalho "Geologia na Escola: Recursos Didáticos e Trabalho de Campo Interdisciplinar” 
é uma dissertação de Mestrado em Geologia, pela Universidade Federal do Rio de Janeiro na qual a autora trás os conceitos de Geociências na perspectiva do Ensino Fundamental II, sob o olhar interdisciplinar (disciplinas de Geografia e Ciências Naturais), onde os conceitos de Geologia são apresentados e discutidos por meio de oficinas voltadas para professores das referidas disciplinas. Trata-se de um momento voltado à formação de professores. Dentre as atividades lúdicas elaboradas, destaca-se a construção de um quebra-cabeças sobre Atmosfera, além de um passeio no Parque Estadual da Pedra Branca, Rio de Janeiro/RJ.

O trabalho "Jogo CICLO DAS ROCHAS: um Recurso Lúdico para o Ensino de Geociências" é uma dissertação derivada do Mestrado em Ensino e História de Ciências da Terra, da Universidade Estadual de Campinas (Unicamp), na qual o autor aborda os conceitos sobre Ciclo das Rochas a partir de um Jogo Didático de Tabuleiro aplicado a estudantes do Curso de Ciências da Terra, na Unicamp. O jogo apresenta diferentes níveis de dificuldade, onde os jogadores vão avançando nas casas por meio de perguntas e informações sobre as rochas. Trata-se, portanto, de uma atividade lúdica que apresenta aspectos interdisciplinares por conta da natureza dos conceitos e dos sujeitos de pesquisa.

O trabalho "Projeto GEO-ESCOLA: Recursos computacionais de apoio ao Ensino de Geociências nos Níveis Fundamental e Médio" deriva também do Programa de Pós-Graduação em Ensino e História de Ciências da Terra, Unicamp. Discute a articulação entre os conceitos de Geologia com as Tecnologias da Educação. O projeto propõe modelos computacionais a serem utilizados por professores e estudantes da Educação Básica, onde são apresentados mapas geológicos do estado de São Paulo.

O trabalho "Possibilidades de Alfabetização Cartográfica a partir de Jogos e Sensoriamento Remoto", desenvolvido no Mestrado em Ensino e História de Ciências da Terra e publicado na Revista Terrae Didatica, aborda os conceitos de Geologia a partir de jogos, como quebra-cabeças, dominó e jogo da memória. Os autores destacam o potencial do lúdico para a Alfabetização Cartográfica e Sensoriamento Remoto, onde são despertados o interesse do estudante pelo conteúdo e desenvolver suas habilidades.

O trabalho "Proposta Metodológica para Aprendizagem de Geociências na Educação Básica” foi realizado na Universidade Católica do Rio
Grande do Sul (Porto Alegre/RS) e foi publicado na Revista Experiências no Ensino de Ciências. Aborda o uso das TIC's para o ensino de Sensoriamento Remoto e o Geoprocessamento no Ensino Fundamental II e Médio, numa perspectiva interdisciplinar (Geografia e Ciências Naturais). Os autores conduzem oficinas de formação continuada com professores, nas quais apresentam alternativas tecnológicas para despertar o interesse e promover a diversão em sala (característica lúdica).

O trabalho "Trilha da Vida em Salinas: uma Ferramenta Lúdica no Ensino de Ciências e na Construção de Conceitos Científicos ligados à Produção Agrícola Local", foi realizado na Universidade Federal de Ouro Preto (Ouro Preto/MG), e publicado na Revista Experiências no Ensino de Ciências. Os autores propõem um Jogo Didático para o estudo de técnicas agrícolas para estudantes do Ensino Fundamental II e Médio, numa perspectiva (Geografia, Ciências Naturais, História), no qual os conceitos de Geologia foram utilizados para estudo do solo. O trabalho foi importante para o desenvolvimento de habilidades vinculadas ao manejo e preparo da terra, para despertar do interesse dos estudantes e para o divertimento.

O trabalho "Proposta de uma Sequência Didática para o Ensino do Tema "Estações do Ano" no Ensino Fundamental" foi realizado na Universidade Federal de São Carlos (São Carlos/SP) e publicado na Revista Ensaio - Pesquisa em Educação em Ciências. Os autores apresentam uma sequência didática aplicada com alunos do Ensino Fundamental II na disciplina de Ciências Naturais, onde foi estudado o conteúdo de Estações do Ano. Essa sequência é composta por diversos tipos de metodologia, dentre elas ressalta-se aquelas com características lúdicas: jogo didático e dramatização (mini-teatro).

Todos os trabalhos identificados estão de acordo com a definição de Geociências proposta nesta pesquisa. A valorização do conhecimento do Sistema Terra e sua dinâmica estão contemplados nas propostas apresentadas nos trabalhos identificados, onde o aspecto lúdico pode ser observado de forma nítida ou de maneira mais sucinta.

Todavia, considerando a pequena quantidade inicial de trabalhos identificados neste levantamento e analisando apenas sete que possuem tendência para o Ensino de Geociências, pode-se afirmar que existe uma carência de propostas que contemplem o tema, e que se apresentem possibilidade de utilização pelo professor da Educação Básica em suas 
aulas. Além disso, nenhum dos trabalhos analisados decorre da experiência docente, ou seja, não foi escrito por professores que implementaram o lúdico em suas aulas para o ensino de conceitos de Geociências. Isto mostra que estes profissionais possivelmente não apresentam segurança para propor novas maneiras (lúdicas ou não) de mediar o conteúdo em sala.

\section{Conclusões}

A pesquisa permitiu a identificação de 349 publicações com foco voltado para o trabalho lúdico no Ensino de Ciências. Desse número, apenas sete ( $2 \%$ do total) apresentam possibilidades para o estudo de conceitos de Geociências na Educação Básica. Torna-se importante ressaltar que o número representa uma realidade para o Ensino de Ciências (da Natureza), ou seja, são trabalhos pensados para o estudo dos conceitos de Geociências.

O número pode ser considerado uma evidência da carência apresentada pelo Ensino de Ciências, uma vez que existem poucas publicações vinculadas ao estudo de conceitos de Geociências. Isso pode justificar, em parte, o motivo de professores de Ciências Naturais (Ensino Fundamental), Química, Física e Biologia, não abordarem de forma efetiva os conceitos de Geologia em suas aulas, ainda que estes estejam presentes nas ementas das disciplinas.

Assim, levando em consideração a categorização dos trabalhos e a análise feita a partir da leitura das publicações vinculadas ao estudo de conceitos de Geociências (no intervalo considerado na pesquisa) é possivel concluir que a literatura científica desta área de conhecimento precisa de mais pesquisa que contemple as disciplinas de Física e Ciências Naturais (Ensino Fundamental) e que gerem novos dados, principalmente que tenham origem em grupos de pesquisa ou em salas de aula do Norte e Centro-Oeste do país. Novas frentes de pesquisa podem trazer novas modalidades lúdicas (não somente Jogo Didático), o que pode ser uma grande contribuição para esta temática.

\section{Referências}

Araujo, C. 2010. Geologia na Escola: Recursos Didáticose Trabalho de Campo Interdisciplinar. Rio de Janeiro: UFRJ. 138p. (Dissert. Mestrado).

Barbosa, R. 2003. Projeto Geo-Escola: Recursos computacionais de apoio ao Ensino de Geociências nos Níveis Fundamental e Médio. Campinas: Unicamp. 131p. (Dissert. Mestrado).

Benevenuti A., Benevenuti A., Hofmann A.A., Brazil F., Marques I., Fuhrmann L R., Silveira M.C., Santaiana R.S.2013. O Lúdico na prática pedagógica. Curitiba: InterSaberes. 210p.

Breda T.V., Picanço J.L., Zacharias A.A. 2012. Possibilidades de Alfabetização Cartográfica a partir de Jogos e Sensoriamento Remoto. In: Revista Terrae Didatica, 9:41-48. URL: https://www.ige. unicamp.br/terrae/V9/PDFv9/Thiara.pdf. Acesso 05.11.2017.

Carneiro C.D.R., Toledo M.C.M.de, Almeida F.F.M.de. 2004. Dez motivos para a inclusão de temas de Geologia na Educação Básica. In: Rev. Bras. Geoc, 34(4):553-560. URL: http://ppegeo. igc.usp.br/index.php/rbg/article/view/9787/9135. Acesso 30.10.2017.

Carneiro C.D.R., Lopes O.R. 2009. O jogo "Ciclo das Rochas" para ensino de Geociências. Rev. Bras. Geoc., 39(1):30-41. URL: http://ppegeo.igc.usp. br/index.php/rbg/article/view/7627/7054. Acesso 21.11.2017.

Ferreira L.H.; Hartwig D.R.; Oliveira R.C. 2010. Ensino Experimental de Química: Uma abordagem investigativa contextualizada. In: Química Nova na Escola, 32:101-1016.

Fourez G. Crise no ensino de ciências? 2003. In: Revista Invest. Ens. Ciênc., 8(2):109-123.

Frodeman R. 1995. Geological reasoning: Geology as an interpretive and historical science. Geol. Soc. Am. Bull., 107:960-968.

Frodeman R. 2010. O raciocínio geológico: a geologia como uma ciência interpretativa e histórica. Terrce Didatica, 6(2):85-99. URL: < http://www.ige. unicamp.br/terraedidatica/v6_2/v6_2_A4.html. Acesso 21.11.2017.

Guimarães C.C. 2009. Experimentação no Ensino de Química: caminhos e descaminhos rumo à aprendizagem significativa. In: Química Nova na Escola, 31(3):198-202.

Kishimoto T.M. 2007. Jogo, brinquedo, brincadeira e a educação. São Paulo: Cortez Ed. 183p.

Lahm R.A, Henriques A., Santos Jr., D. N., Borges R. M. R., 2010. Proposta Metodológica para Aprendizagem de Geociências na Educação Básica. In: Exper. Ens. Ciências, 5(2):7-16.

Luckesi C.C. 1982. Independência e inovação em tecnologia educacional.: Tecnologia Educacional, 15(71-72):55-64.

Oliveira N. 2009. Atividades de experimentação investigativas lúdicas no ensino de química: um estudo de caso. Goiania: UFG. 147p. (Tese Dout.).

Potapova M.S. 1968. Geology as an historical science of nature. In: Interaction of science in the study of the earth. Moscow: Progress. 1:117-126. Potapova M.S. 2008. Geologia como uma ciência históri- 
ca da natureza. Terre Didatica, 3(1):86-90. URL: http://www.ige.unicamp.br/terraedidatica/v3/ v3 a7.html. Acesso 22.11.2017.

Rau M.C.T.D. 2011. A Ludicidade na Educação: Uma atitude pedagógica. Curitiba: Ibpex. 284p.

Siqueira L.G., Franco M.A.M., Moreira L.M. 2016. Trilha da Vida em Salinas: uma Ferramenta Lúdica no Ensino de Ciências e na Construção de Conceitos Científicos ligados à Produção Agrícola Local. Exper. Ens. Ciênc., 11(1):88-100. URL: http://if.ufmt.br/eenci/artigos/Artigo_ID302/v11_ n1_a2016.pdf. Acesso em 30.10.2017.

Soares M.F.B. 2013. Jogos e atividades lúdicas para o Ensino de Química: Teoria, Métodos e Aplicações. In: Enc. Nac. Ens. Química, 14, 2013 (XIV ENEQ). Anais... SBQ. V. 1, p. 1-12.
Taxini C.L., Puga C.C.I., Silva C.S.F., Oliveira R.R. 2012. Proposta de uma Sequência Didática para o Ensino do Tema "Estações do Ano" no Ensino Fundamental. Ens. Pesqui. Educ. Ciênc.14(1):81-97. URL: http://dx.doi. org/10.1590/1983-21172012140106. Acesso 30.10.2017.

Teixeira D.M. 2016. Contribuições dos jogos didáticos na formação inicial de professores de química da Universidade Estadual de Santa Cruz. Univ. Est. Santa Cruz. 143p. (Dissert. Mestr.).

Veiga I.P.A. 2006. Projeto político-pedagógico da escola: uma construção possível. Campinas, SP: Papirus. 26p.

Villardi R., Oliveira E.S.G. 2005. Tecnologia na educação: uma perspectiva sócio-interacionista. Rio de Janeiro: Dunya. 127p.

Resumo: As atividades lúdicas são metodologias de ensino que vêm conquistando espaço no Ensino de Ciências; proporcionam um estudo dos conceitos de forma mais dinâmica e prazerosa. Todavia, quando se trata dos conceitos de Geociências as dificuldades apresentadas pelos professores de Ciências Exatas e Biológicas acabam por fazer com que estes profissionais não elaborem propostas vinculadas a tais conceitos. 0 objetivo deste trabalho é, portanto, identificar na literatura científica do Ensino de Ciências as principais tendências de pesquisas com características lúdicas que possuam como foco os conceitos de Geociências. Para isso, foi realizada uma busca em: (a) banco de Teses e Dissertações da Coordenação de Aperfeiçoamento de Pessoal de Nível Superior (CAPES), e (b) periódicos vinculados ao Ensino de Ciências. Como resultado, foi possível observar que as publicações lúdicas no Ensino de Ciências apresentam as seguintes tendências: (1) A maior parte das publicações tem origem na região Sudeste, principalmente nos estados de Rio de Janeiro e São Paulo; (2) 0 número de publicações é crescente a partir do ano de 2014, com ponto forte no ano 2016; (3) a maior área de conhecimento a apresentar trabalhos é Química, seguida de Biologia; (4) as publicações são, em sua maior parte, experiências realizadas no Ensino Médio, e (5) a modalidade lúdica preferencial é "Jogo Didático".

Palavras-Chaves: Lúdico, Geociências, Ensino de Ciências. 